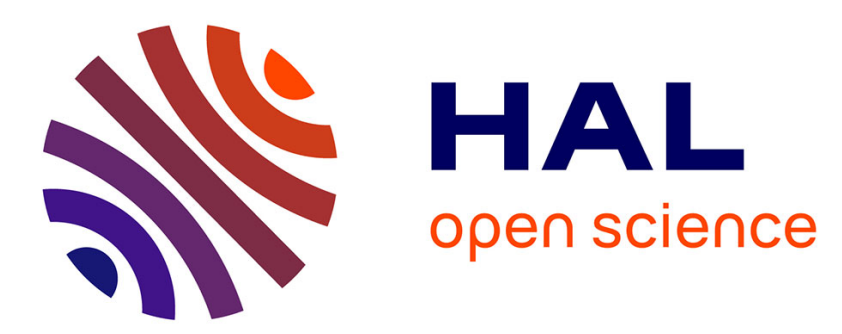

\title{
Estimation of convection speed in underexpanded jets from schlieren pictures
}

Thomas Castelain, Romain Gojon, Bertrand Mercier, Christophe Bogey

\section{To cite this version:}

Thomas Castelain, Romain Gojon, Bertrand Mercier, Christophe Bogey. Estimation of convection speed in underexpanded jets from schlieren pictures. 22nd AIAA/CEAS Aeroacoustics Conference, May 2016, Lyon, France. pp.1-14, 10.2514/6.2016-2984 . hal-02352884

\section{HAL Id: hal-02352884 \\ https://hal.science/hal-02352884}

Submitted on 19 Apr 2021

HAL is a multi-disciplinary open access archive for the deposit and dissemination of scientific research documents, whether they are published or not. The documents may come from teaching and research institutions in France or abroad, or from public or private research centers.
L'archive ouverte pluridisciplinaire HAL, est destinée au dépôt et à la diffusion de documents scientifiques de niveau recherche, publiés ou non, émanant des établissements d'enseignement et de recherche français ou étrangers, des laboratoires publics ou privés. 


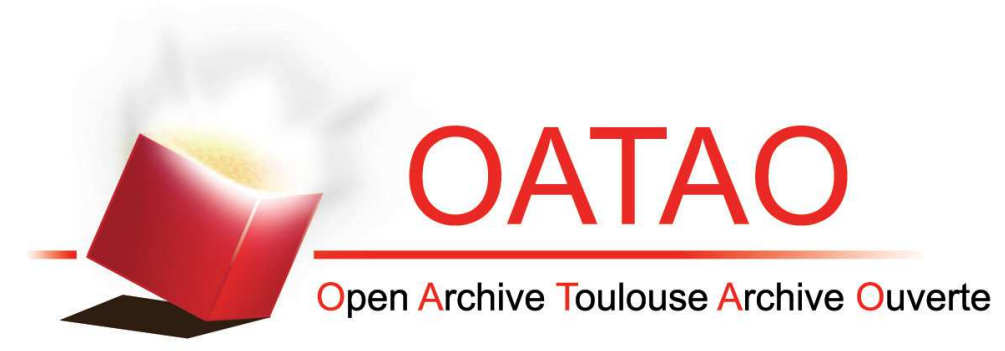

\section{Open Archive Toulouse Archive Ouverte (OATAO)}

OATAO is an open access repository that collects the work of some Toulouse researchers and makes it freely available over the web where possible.

This is an author's version published in: https://oatao.univ-toulouse.fr/27038

Official URL:https://doi.org/10.2514/6.2016-2984

\section{To cite this version :}

Castelain, Thomas and Gojon, Romain and Mercier, Bertrand and Bogey, Christophe Estimation of convection speed in underexpanded jets from schlieren pictures. (2016) In: 22nd AIAA/CEAS Aeroacoustics Conference, 30 May 2016 1 June 2016 (Lyon, France).

Any correspondence concerning this service should be sent to the repository administrator: tech-oatao@listes-diff.inp-toulouse.fr 


\title{
Estimation of convection speed in underexpanded jets from schlieren pictures
}

\author{
Thomas Castelain ${ }^{1,2, *}$, Romain Gojon ${ }^{1, \dagger}{ }^{1}$, Bertrand Mercier ${ }^{1, \ddagger} \&$ Christophe Bogey ${ }^{1, \S}$ \\ 1- $\quad$ Univ Lyon, Laboratoire de Mécanique des Fluides et d'Acoustique, UMR 5509, \\ École Centrale de Lyon, 36 av. Guy de Collongue, F-69134 Ecully Cédex, France \\ 2- Univ Lyon, Université Lyon 1, 43 Bd du 11 Novembre 1918, F-69622 Villeurbanne Cédex, France
}

\begin{abstract}
In this paper, the quality of the estimation of the convection velocity in jet shear layers using schlieren pictures is investigated. The aim is to discuss whether the convection velocity is likely to be biased if determined from schlieren images obtained at a high frame rate, as in previous experiments using the phase shift method. For this, a numerical procedure is developed in order to generate schlieren-like images on the basis of simulation data, and applied to the results provided by the large-eddy simulation (LES) of an underexpanded round jet at an ideally expanded Mach number of 1.56. The results obtained from the schlieren pictures are compared with those obtained directly from the LES density fields. It is notably found that the location of the maximum of gray level fluctuations in the schlieren pictures corresponds well to that of the maximum of density fluctuations, and that the convection velocity estimated for low frequencies using schlieren pictures is underestimated for small separation distances between the two points used for the phase shift calculation.
\end{abstract}

\section{Introduction}

In aeroacoustics models for supersonic jet noise, ${ }^{1,2}$ one input parameter related to the flow itself is the convection velocity of the turbulence in the jet shear layer. In recent years, different experimental methods ${ }^{3-8}$ were applied to measure the convection velocity in high-speed jets, and when possible, its dependency on the frequency. In several studies, a schlieren system is set up together with imaging optics so that the light intensity in the image plane of the jet is measured by use of two combined photodiodes ${ }^{6,9,10}$ or of a high speed camera. ${ }^{7,8}$ The data analysis seeks for instance at determining the phase difference between two signals from a couple of sensors. This phase shift is related (at least partly) to convection and hence, an estimate of the convection velocity is obtained as soon as the distance between the two sensors is known. Conventional schlieren imaging is known to provide pictures whose contrast results from the integration of all the light beam perturbations from the light source to the sensor. Sharp-focusing systems ${ }^{11,12}$ can provide two-dimensional slices of a flow, with a focus depth depending on the experimental apparatus chosen. In practical, ${ }^{11}$ the focus depth is of the order of the diameter of the Lab-scale round supersonic jets.

This paper is an attempt to determine if the characteristics of conventional schlieren imaging (space integration over the light path, sensitivity to gradients of density rather than the density itself) can affect the convection velocity estimation.

In this purpose, experimental schlieren images of an underexpanded jet at $M_{j}=1.50$ are first exploited. To estimate the convection velocity, the phase shift method is applied by using two time signals extracted from the time-series of schlieren pictures acquired at a high frame rate. The distance between the two points is varied and its influence on the convection velocity estimation is highlighted. To go further, a study based on synthetic schlieren pictures processed from 3-D data obtained by Large Eddy Simulation (LES) of a round underexpanded $M_{j}=1.56$ jet is presented. These pictures, obtained by using a specific numerical procedure,

\footnotetext{
*Assistant Professor, Université Lyon 1, France

${ }^{\dagger} \mathrm{PhD}$, currently Post-doctoral student at KTH Royal Institute of Technology, Sweden

$\ddagger$ PhD student, Univ Lyon, École Centrale de Lyon

$\S$ CNRS Research Scientist, AIAA Senior Member and Associate Fellow
} 


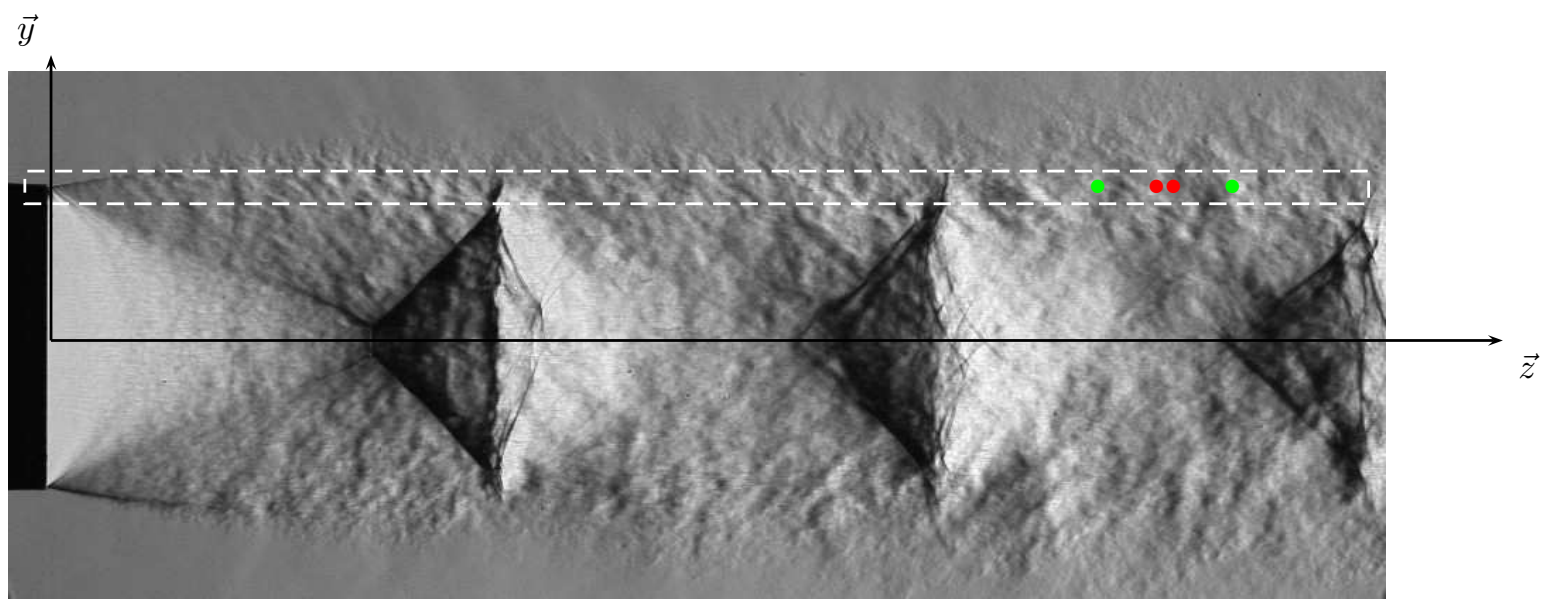

Figure 1. Single-frame schlieren image for $M_{j}=1.50$. The white dashed area indicates the field of view recorded at a high frame rate; the symbols indicate the typical locations where the data are extracted to apply the convection velocity estimation procedure by two-point phase difference, the axial distance between the two points ranging from $\bullet 2 \delta_{z}=8 \mathrm{px}\left(\right.$ ie $\left.2 \delta_{z} / D=\mathbf{0 . 0 5 5}\right)$ to $\bullet 2 \delta_{z}=64 \mathrm{px}\left(\right.$ ie $\left.2 \delta_{z} / D=0.44\right)$

are validated against reference quasi-analytic solutions. The convection velocity is estimated again using the phase shift method, as for the experiments. The results are compared with those derived from the same methodology applied to the density field - the raw data provided by the numerical simulations. The analysis aims to show that the spacing between the two points affects the convection velocity estimation from the schlieren pictures. Before presenting these results, the parameters and methods used in the experiments and in the simulation are first given.

\section{Jets parameters and methods used}

\section{A. Experimental set-up}

The experiments were done in the $10 \mathrm{~m} \times 8 \mathrm{~m} \times 8 \mathrm{~m}$ anechoic room of the Centre Acoustique, Laboratoire de Mécanique des Fluides et d'Acoustique at École Centrale de Lyon. A contoured convergent nozzle of diameter $D=38 \mathrm{~mm}$, is continuously supplied by a centrifugal compressor in unheated dry air. The wall static pressure is measured 15 nozzle diameters upstream of the exit. Stagnation pressure is then retrieved from the wall static pressure value through the estimate of the local Mach number in the measurement section. The supersonic jet studied here is associated to an ideally expanded Mach number $M_{j}=1.50$ and a total temperature around $30^{\circ} \mathrm{C}$. The associated Reynolds number based on the fully expanded velocity and the nozzle diameter $D$ is $R e_{D}=2 \times 10^{6}$. A representative view of the choked jet is provided in Figure 1 using a conventional Z-type schlieren system. The imaging system used in the following consists of a continuous Cree XHP LED light source, a knife edge set perpendicular to the jet axis, and two $f / 8,203.2$-mm-diam parabolic mirrors arranged so that the off-axis setting is limited to $10 \mathrm{deg}$. The schlieren images are recorded by a high-speed Phantom V12 CMOS camera whose frame rate is set to $430769 \mathrm{~Hz}$, with an exposure time of $1.87 \mu \mathrm{s}$. The total length of one recording is $1.21 \mathrm{~s}$ which corresponds to 521472 successive images. To ensure the high acquisition frame rate, the recorded image area is limited to a $640 \mathrm{px} \times 16 \mathrm{px}$ region centered on the upper jet shear layer. Using appropriate collimating optics, the image resolution is set to $0.261 \mathrm{~mm} / \mathrm{px}$. With these settings, the field of view corresponds to 4.4D in the longitudinal direction and to 0.11D in the radial direction, as can be observed in the typical image depicted in Figure 1. In this Figure the location of the couple points used for the estimations of convection velocity, arbitrary placed on the nozzle lipline and in the middle of the third shock cell, is also represented. This position is noted $\mathbf{x}_{0}$ in the following. Similar results as those presented in section III were also obtained for other locations at various distances to the nozzle exit along the lipline.

\section{B. Numerical simulation of a round underexpanded $M_{j}=1.56$ jet}

A supersonic round jet has been computed by solving the unsteady compressible Navier-Stokes equations using low-dispersion and low-dissipation schemes ${ }^{13-15}$. Further details on the simulation are provided in a previous paper. ${ }^{16}$ The jet is underexpanded, and is characterized by a Nozzle Pressure Ratio of NPR = $P_{r} / P_{\mathrm{amb}}=4.03$, where $P_{r}$ is the stagnation pressure and $P_{\mathrm{amb}}$ is the ambient pressure. The fully expanded 
Mach number is $M_{j}=1.56$, the exit Mach number $M_{e}=1$, and the Reynolds number is $R e_{D}=5 \times 10^{4}$. The mesh contains 400 million points, with mesh spacings allowing acoustic waves with Strouhal numbers up to $S t_{D}=5.6$ to be well propagated. The 3 -D flow density of the simulation is recorded at a sampling frequency of $S t_{D}=6.4$, over a volume defined in cylindrical coordinates by 200 points in the radial direction with a maximum radial position of $r=1.5 D, 512$ points in the azimuthal direction covering $2 \pi$ and 491 points along the jet axis ranging up to $z=5.15 \mathrm{D}$. In the following, a light beam along the Cartesian $\vec{x}$ axis is supposed to go through the underexpanded jet, as represented in the figure 2. The equations governing refractions of the light beam due to the variations in the flow density, derived from Fermat's principle, are provided in the next section.

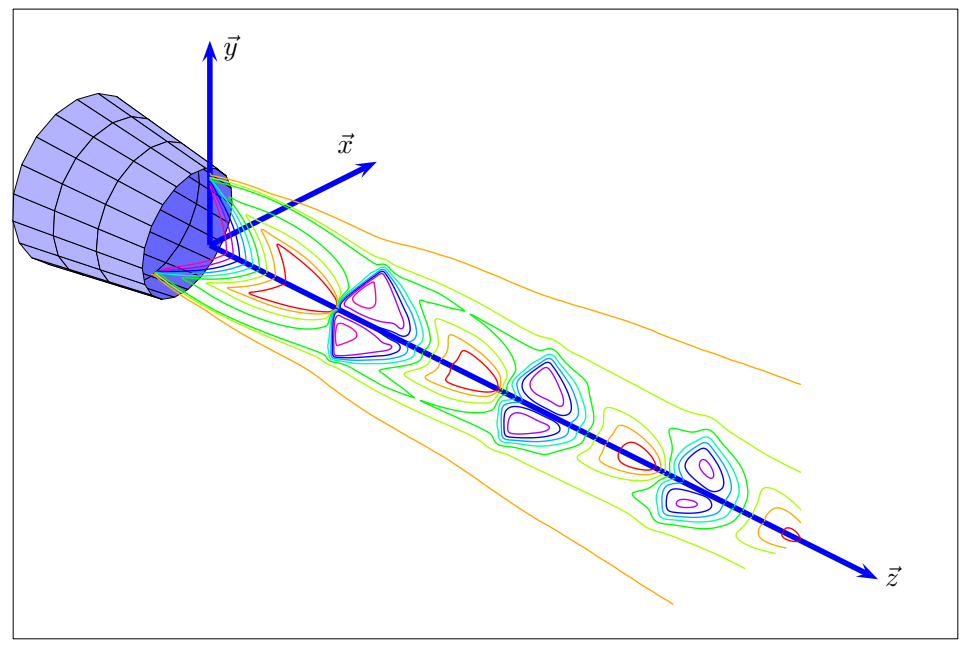

Figure 2. Time-averaged density field of an underexpanded, $M_{j}=1.56$ and $R e_{D}=5 \times 10^{4}$, jet, obtained by simulation. ${ }^{16}$ Isovalues of density from 1 to $2.6 \mathrm{~kg} . \mathrm{m}^{-3}$, by step $0.2 \mathrm{~kg} . \mathrm{m}^{-3}$, are presented.

\section{Two-point method for convection velocity estimation}

The convection velocity may be estimated from the phase lag between two signals representative of the flow, recorded at two different points in the jet shear layer. These could be density signals, ${ }^{3}$ velocity signals ${ }^{4,5}$ or optical signals depending on density gradients. ${ }^{6,7,9,10}$ For one physical position $\mathbf{x}$ within the flow, let $g(\mathbf{x}, t)$ be the signal measured by the sensor used at the time $t$. Calling $\mathcal{G}(\mathbf{x}, \mathrm{f})$ the Fourier transform of $g(\mathbf{x}, t)$ and taking the $\mathbf{x}$ location as a reference point, the cross-spectrum $\mathcal{G}_{\delta z}(\mathbf{x}, f)$ between the signal coming from $\mathbf{x}+$ $\delta z \vec{z}$ and the one coming from $\mathbf{x}-\delta z \vec{z}$ is computed using :

$$
\mathcal{G}_{\delta z}(\mathbf{x}, f)=\mathcal{G}(\mathbf{x}+\delta z \vec{z}, f) \times \mathcal{G}^{\star}(\mathbf{x}-\delta z \vec{z}, f)
$$

where the ${ }^{\star}$ denotes complex conjugate.

The estimation of the convection velocity $U_{c}$ is derived from the phase $\Phi_{\delta z}(\mathbf{x}, f)$ of the cross-spectrum $\mathcal{G}_{\delta x}(\mathbf{x}, f)$ using :

$$
U_{c}(\mathbf{x}, f)=2 \pi f \frac{2 \delta z}{\Phi_{\delta z}(\mathbf{x}, f)}=\frac{4 \pi f \delta z}{\Phi_{\delta z}(\mathbf{x}, f)}
$$




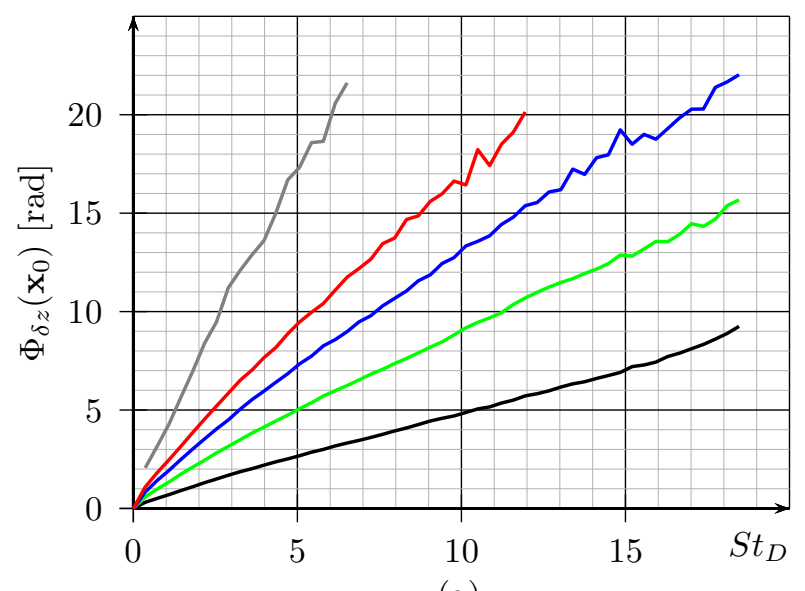

(a)

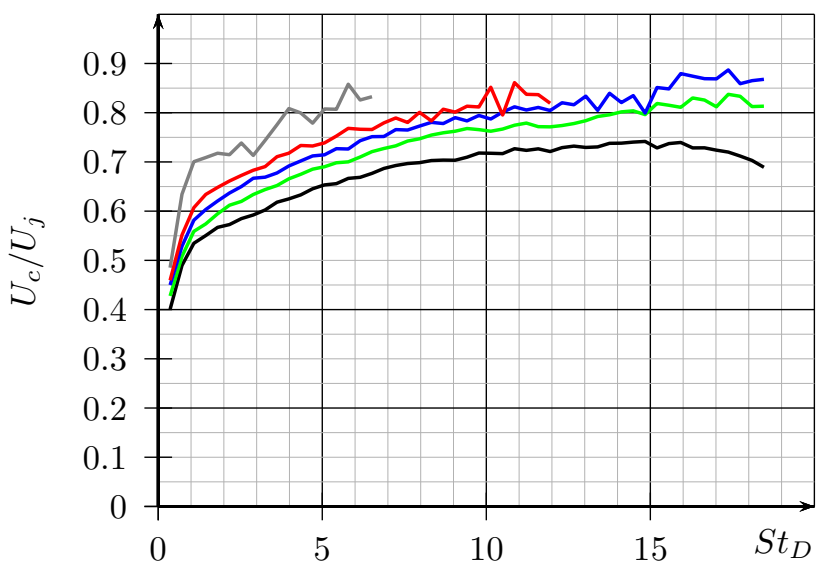

(b)

Figure 3. (a) Phase lag $\Phi_{\delta z}\left(\mathrm{x}_{0}\right.$ for different couples of points centered on $x_{0}:-\delta_{z}=4 \mathrm{px} ;=\delta_{z}=8 \mathrm{px}$; $\delta_{z}=12 \mathrm{px} ;-\delta_{z}=16 \mathrm{px} ;-\delta_{z}=32 \mathrm{px}$, (b) Estimation of the non-dimensioned convection velocity $U_{c} / U_{j}$ for the same couples of points centered on $x_{0}$

\section{Experimental results for an underexpanded $M_{j}=1.50$ jet}

High-speed schlieren cinematography has previously been proposed using the Cranz-Schardin principle, ${ }^{17}$ which allowed for up to 4 consecutive schlieren images with a minimum time separation of $1 \mu \mathrm{s}$. The idea proposed here is to use the tremendous capacities of high-speed digital cameras to construct time signals extracted from a sequence of several hundreds of thousands consecutive images. Grayscale time signals from the schlieren images would then be used as the input signals for cross-spectrum computation as in (1). Because of the knife-edge orientation, orthogonal to the jet axis in this experimental study, the signal will be denoted as $g_{z}$, where the subscript $z$ is used to indicate that the contrast in these experimental schlieren images is due to density gradients along the $\vec{z}$ axis.

In this study, the experimental signals were segmented into 9997 blocks of 104 points, obtained using a $50 \%$ overlap, to compute the mean cross-spectrum of which the phase is extracted. Considering in Figure $3(\mathrm{a})$ the evolution of the phase $\Phi_{\delta z}\left(\mathbf{x}_{0}\right)$ as a function of the Strouhal number $S t_{D}=f D / U_{j}$ based on the nozzle diameter and the perfectly expanded jet velocity $U_{j}$, one notices that the phase delay monotonically increases with $S t_{D}$ for the tested values of $\delta z$. For the two largest values of $\delta z$, the phase shift is limited to approximately 20 radians because noise limits the estimation accuracy for larger values. The convection velocity, estimated using (2) and given in Figure 3(b), follows the behavior obtained in previous studies, by globally increasing monotonically with $S t_{D}$ and reaching values around $0.7 U_{j}$ or $0.8 U_{j}$. Nevertheless, as pointed out earlier, ${ }^{3,6,7}$ the distance $\delta z$ between the probes affects the estimation of the convection velocity over the whole range of $S t_{D}$.

This influence must be taken into account also because the differences in $U_{c}$ are noticeable (around $10 \%$ ) for the smallest separations $2 \delta z=8 \mathrm{px}$ and $16 \mathrm{px}$ (respectively $2 \delta z / D=0.055$ and 0.110 , which corresponds to $2 \delta z=2.1 \mathrm{~mm}$ and $4.2 \mathrm{~mm})$, that are of the order of the local shear layer momentum thickness $\left(\delta_{\theta} \approx 3.3\right.$ $\mathrm{mm}$ for $\mathbf{x}_{0}=(3.8 D, 0.5 D)$ as mentioned in Fig 3.42 of a previous study $\left.{ }^{7}\right)$ and thus small with respect to the jet diameter $D$. Panda ${ }^{3}$ reminds us that the distortion of the eddies that occurs over the separation distance also influences in the phase, thus there should be at least one part of the error in the estimation of the convection velocity that comes from the phase lag method itself. This effect is noticeable in Panda ${ }^{3}$ for the case of a round $M_{j}=0.95$ jet; for a separation between the two probes of around one jet diameter $D$ at $S t_{D}=1.6$, the lack of coherence between the two experimental signals induces errors in the phase estimate. For smaller probes separations, the linear dependency of the cross-spectral phase with the probes separation is remarkable, which indicates that the separation between the probes induces only a small, if any, bias in the convection velocity estimate.

To summarize, probes separation in high-speed conventional schlieren imaging affects the convection velocity estimation, as it is also the case in previous studies ${ }^{3,6}$ where it is proposed to calculate this quantity by averaging over multiple separation points. Considering the large difference between these estimations 
for high-speed conventional schlieren imaging, such an averaging could lead here to significant errors in the convection velocity calculation. Thus, the approach proposed hereafter consists in determining whether there is a range of appropriate probe separations for which the error in the convection velocity can be minimized. This approach is based on numerical simulations and treatments of these data that are described in the following sections.

\section{Synthetic schlieren images generation and analysis}

In this section, the aim is to determine the similarities between flow characteristics and those derived from schlieren imaging. For this, synthetic schlieren images are generated from a reference 3-D density field obtained by the simulation described in section II.B. Results obtained the quantitative analysis of these schlieren images are compared to the real characteristics of the flow field. A brief description of the basics of schlieren imaging is first given, and the numerical procedure used to generate the schlieren images on the basis on numerical data is presented. The main results of the comparisons are finally provided.

\section{A. Schlieren imaging}

A light beam is supposed here to propagate along the $\vec{x}$ axis when the medium is homogeneous. In the presence of perturbations in the medium making it optically inhomogeneous, the light path is given by the following system of differential equations ${ }^{18}$

$$
\left\{\begin{array}{l}
\frac{\mathrm{d}^{2} y}{\mathrm{~d}^{2} x}=\frac{1}{n}\left(1+\left(\frac{\mathrm{d} y}{\mathrm{~d} x}\right)^{2}+\left(\frac{\mathrm{d} z}{\mathrm{~d} x}\right)^{2}\right)\left(\frac{\partial n}{\partial y}-\frac{\mathrm{d} y}{\mathrm{~d} x} \frac{\partial n}{\partial x}\right) \\
\frac{\mathrm{d}^{2} z}{\mathrm{~d}^{2} x}=\frac{1}{n}\left(1+\left(\frac{\mathrm{d} y}{\mathrm{~d} x}\right)^{2}+\left(\frac{\mathrm{d} z}{\mathrm{~d} x}\right)^{2}\right)\left(\frac{\partial n}{\partial z}-\frac{\mathrm{d} z}{\mathrm{~d} x} \frac{\partial n}{\partial x}\right)
\end{array}\right.
$$

The deflection of the light beam is usually considered sufficiently small to allow the terms $\mathrm{d} y / \mathrm{d} x$ and $\mathrm{d} z / \mathrm{d} x$ to be negligible with respect to unity. The light path is thus governed by the simplified differential system :

$$
\left\{\begin{array}{l}
\frac{\mathrm{d}^{2} y}{\mathrm{~d}^{2} x}=\frac{1}{n} \frac{\partial n}{\partial y} \\
\frac{\mathrm{d}^{2} z}{\mathrm{~d}^{2} x}=\frac{1}{n} \frac{\partial n}{\partial z}
\end{array}\right.
$$

The difference between the solutions of the two differential equation systems (3) and (1) was evaluated in the case of time-averaged data. The difference was found to be so small with respect to the spatial resolution of the generated images that the system (1) is considered in the following to provide a good representation of the real perturbation of the light path by the density variations inside the jet.

Schlieren imaging consists in focusing the light beam perturbed by the density field tested, on an image plane corresponding to, for example, a CMOS or CCD sensor. Before the sensor, one part of the light beam is cut off by a knife-edge whose orientation determines the link between the image contrast and the physical quantity visualized. Hence, if the knife-edge is aligned with the $\vec{y}$ axis, in other terms orthogonal to the jet axis, the relative intensity change in the recording plane is given by: ${ }^{18,19}$

$$
\frac{\Delta I}{I}=K^{\prime} \int_{-\infty}^{\infty} \frac{\partial \rho}{\partial z} \mathrm{~d} x
$$

in which the refractive index of the tested field is supposed to remain close to unity, and $K^{\prime}$ a constant depending on the optical set-up and the Gladstone-Dale constant linking the refractive index and the gas density.

Similarly, if the knife-edge is aligned with the $\vec{z}$ axis, the relative intensity change in the recording plane thus follows:

$$
\frac{\Delta I}{I}=K^{\prime} \int_{-\infty}^{\infty} \frac{\partial \rho}{\partial y} \mathrm{~d} x
$$


Thus, for a given optical set-up and a given gas, the schlieren images are entirely determined by the integral term in the equations (1) and (2). In the following, the general notation $f$ is chosen for the function to be integrated along $x$, which depends on the orientation of the schlieren knife-edge to be reproduced.

\section{B. Numerical procedure for the computation of synthetic schlieren images}

In the following, $f$ is considered as a function depending on four variables : the time $t$ and three space variables. Moreover, the associated time-averaged function $\bar{f}$ is a function of two space variables only, because in addition to the vanishing of the dependency with $t$, the property of axisymmetry of the jet flow field induces also that $\bar{f}$ does not depend on the azimuthal coordinate.

Thus, the intensity distribution of the schlieren image at the point $\left(z_{0}, y_{0}\right)$ and a given time $t$, assuming a magnification factor equal to unity, is proportional to $\epsilon_{f}\left(z_{0}, y_{0}\right)$ defined as:

$$
\epsilon_{f}\left(z_{0}, y_{0}, t\right)=\int_{-\infty}^{\infty} f\left(r, \theta, z_{0}, t\right) \mathrm{d} x
$$

Moreover, for $\bar{f}$,

$$
\epsilon_{\bar{f}}\left(z_{0}, y_{0}\right)=\int_{-\infty}^{\infty} \bar{f}\left(r, z_{0}\right) \mathrm{d} x
$$

The approach followed here is to reproduce, from density field provided by the simulation described in section B, a set of schlieren images as could be obtained experimentally and to apply the analyses developed for experimental data ${ }^{3,6,7}$ to these 'synthetic' images. There is a need to verify that the 'synthetic' images are correctly generated from the simulation. Moreover there is also a need to develop an efficient method to compute the integral terms in (1) and (2). Thus two different methods to compute these synthetic schlieren images are presented below, from the time-averaged flow field from the simulation.

\section{Reference solution for the time-averaged axisymmetric jet}

Recalling the relation obtained using the axisymmetry property for $\bar{f}$, integrated over a light path parallel to the $\vec{x}$ axis and distant from $y_{0}$ to this axis, as denoted in Figure 4:

$$
\epsilon_{\bar{f}}\left(z_{0}, y_{0}\right)=2 \int_{0}^{\infty} \bar{f}\left(\sqrt{x^{2}+y_{0}^{2}}, z_{0}\right) \mathrm{d} x
$$

with $r=\sqrt{x^{2}+y_{0}^{2}}$. With the variable change in the calculation of $\epsilon_{f}$, as also noticed previously, ${ }^{20}$ one obtains:

$$
\epsilon_{\bar{f}}\left(z_{0}, y_{0}\right)=\int_{y_{0}}^{\infty} \bar{f}\left(r, z_{0}\right) \frac{r}{\sqrt{r^{2}-y_{0}^{2}}} \mathrm{~d} r
$$

As a consequence, the knowledge of the function $\bar{f}\left(r, z_{0}\right)$ for $r \in[0: \infty]$ is sufficient to determine the light intensity distribution over the schlieren image by computing the integral defined in equation (5). This procedure provides a reference solution to which the results of the direct integration of the volume domain are compared.

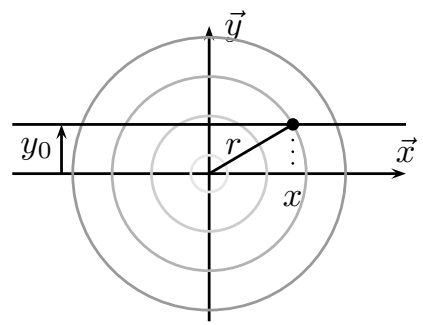

Figure 4. Light beam position (parallel to $\vec{x}$ ) with respect to the axisymmetric isocontours of $\bar{f}$. 


\section{Direct integration over light path}

The direct evaluation of integrals (3) or (4) relies on the data available along the light path, supposed here to be oriented along the $\vec{x}$ axis, as described in Figure 4. On the other hand, the data from the simulation, and thus the values for $f$ (or $\bar{f}$ ), are available over a cylindrical mesh; data interpolation thus needs to be done. A strategy consists in interpolating on a Cartesian mesh the data contained in a $(\vec{x}, \vec{y})$ plane for a given value of $z$, then computing the requested integrals and stepping to the next $(\vec{x}, \vec{y})$ plane. This strategy turns out to be costly because of the 2-D interpolation from polar to Cartesian meshes. Thus, a second strategy consists in performing 1-D data interpolation along the datasets at a given $\theta$. It appears to be around 20 times faster than the first strategy. As illustrated in Figure 5, the data are first interpolated along their radial distribution at the points (in red in Figure 5) located on the light path considered. Then, the distance between two consecutive samples is evaluated, so that the integral given in (3) is approximated by :

$$
\epsilon_{f}\left(z_{0}, y_{0}, t\right)=\int_{-\infty}^{\infty} f\left(r, \theta, z_{0}, t\right) \mathrm{d} x \approx \sum_{k} f\left(\frac{y_{0}}{\sin \left(\theta_{k}\right)}, \theta_{k}, z_{0}, t\right) \Delta x_{k}
$$

with the increment in $x$, noted $\Delta x_{k}$, being computed as :

$$
\Delta x_{k}=\frac{1}{2}\left(x_{(k-1) k}+x_{k(k+1)}\right) \quad \text { where } \quad x_{k(k+1)}=y_{0}\left(\frac{1}{\tan \left(\theta_{k}\right)}-\frac{1}{\tan \left(\theta_{(k+1)}\right)}\right)
$$

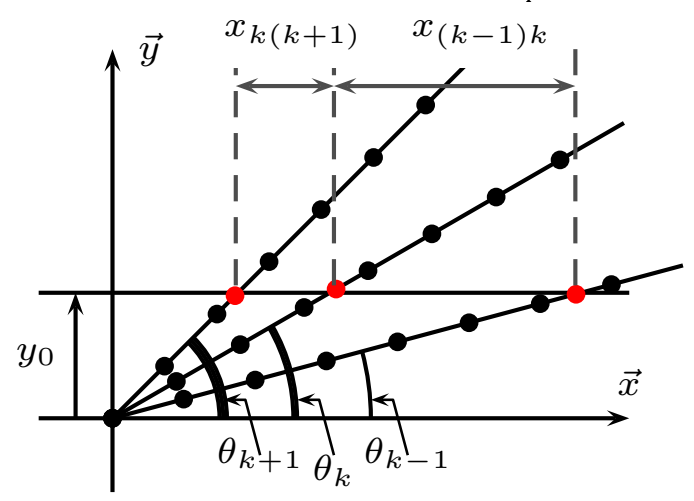

Figure 5. Interpolation method for the computation of $\epsilon_{f}\left(y_{0}, z_{0}\right)$ : the black dots represent the locations where the values of $f$ are known (as a result of the numerical simulation using cylindrical coordinates), the red dots represent the locations where $f$ is interpolated (1-D interpolation in the radial direction at a given $\theta$ ). Three over the 512 values of $\theta$ are represented here.

Some synthetic schlieren pictures obtained using the procedure described above are now presented. In Figure 6 a schlieren picture obtained experimentally ${ }^{7}$ and a synthetic picture based on an instantaneous 3-D density field from the simulation are provided. For the experimental result, the knife-edge was oriented along the jet axis, which is reproduced in the numerical approach by choosing $f \equiv \frac{\partial \bar{\rho}}{\partial y}$. There is a good agreement between the two pictures, both capturing the peculiarities of the underexpanded jet flow. The shock-cell structure as well as coherent structures developing upstream of the nozzle exit and turbulence are clearly visible, similarly to experimental results for underexpanded jets. ${ }^{21,22}$ Differences are noticed in the size of the structures developing in the jet shear layer next to the nozzle exit which can be attributed to differences between the Reynolds numbers in the simulation and the experiment as well as probable differences in the jet exit conditions.

In Figure 7 the synthetic images built from an instantaneous density field obtained by the simulation and from time-averaged data are compared. In particular, the images for the time-averaged 3-D density field, determined as described in section 2, are compared with those obtained by applying equation (5) to the time-averaged density profiles determined by density averaging in the azimuthal direction. The very good match between the two sets of results confirms the ability of the numerical procedure implemented to provide schlieren-like images. Depending on the expression of $f$ in equation (6), and/or equivalently the orientation of the knife-edge in a corresponding experiment, some features of the jet flow are more easily 

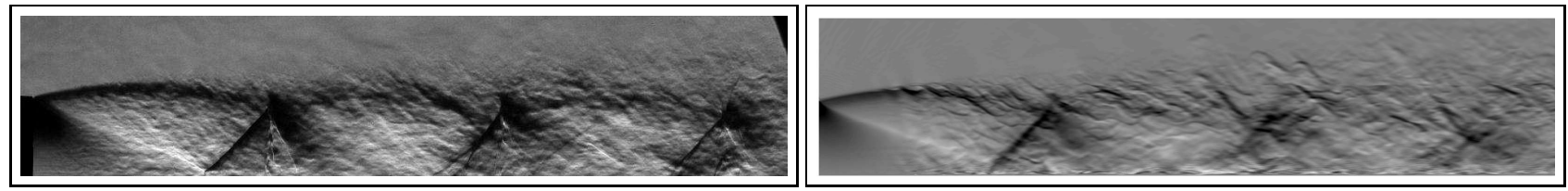

Figure 6. Schlieren images obtained from experiments ${ }^{7}$ at $\operatorname{Re}_{D}=2 \times 10^{6}$ (left) and from the simulation at $R e_{D}=5 \times 10^{4}$ (right).

discernible. For example, the azimuthal correlation of the coherent structures in the first part of the first shock cell is clearly noticeable if the axial density gradient is considered.

Finally, spurious noise very close to the jet axis (bottom of the image in the two upper lines of Figure 7) can be observed, which corresponds to the fact that the approximation proposed in equation (6) is less appropriate for small values of $y_{0}$. Nevertheless, as the aim is to focus hereafter in the region centered around the top shear layer, these limitations are not critical.

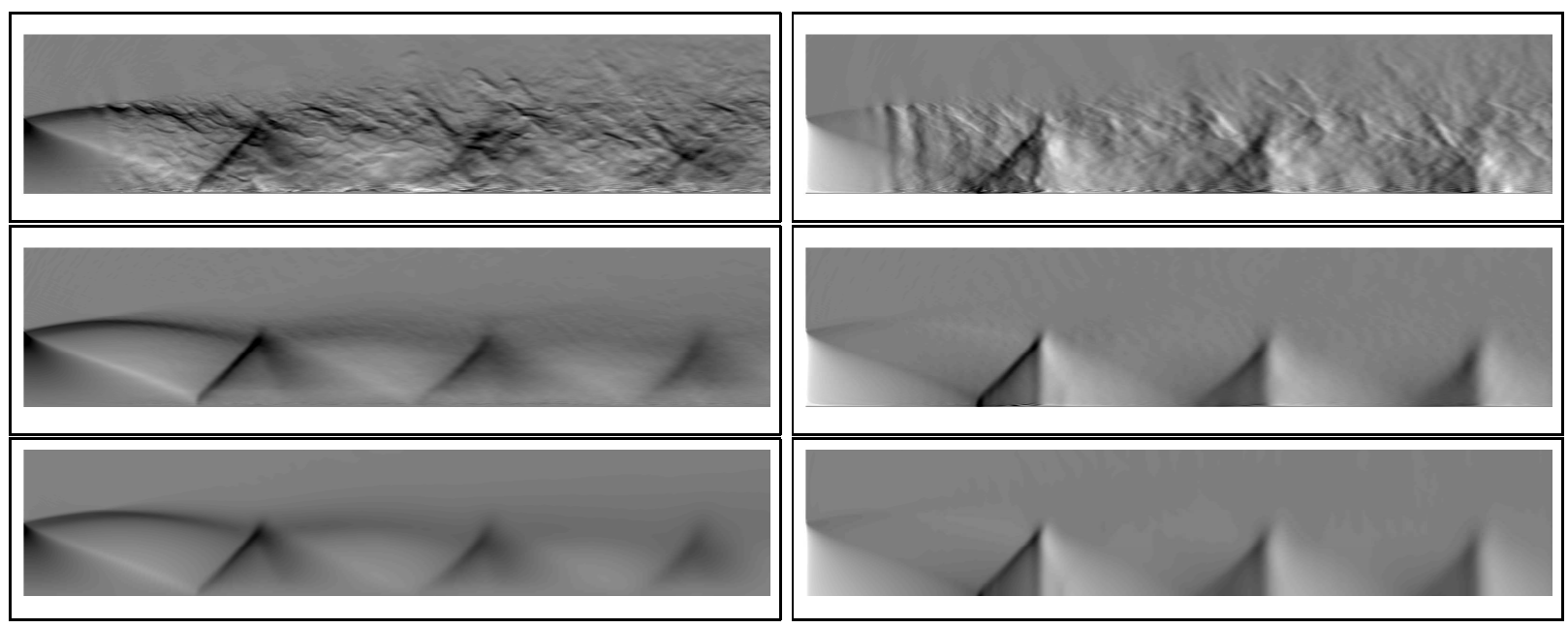

Figure 7. Synthetic schlieren pictures computed using one 3-D flow density field at a given time $t$ (top), the time-averaged 3-D density field (middle) and the mean density profile combined with equation (5) (bottom). Pictures in the left column correspond to $f \equiv \partial \rho / \partial y$, i.e. (virtual) knife-edge oriented along the jet axis, and pictures in the right column correspond to $f \equiv \partial \rho / \partial z$, i.e. knife-edge normal to the jet axis. Only the top-half of the jet is considered

\section{Comparisons of density and schlieren data}

\section{Standard deviation of quantities related to the flow}

On the basis of the density field from the simulation, from which one snapshot is presented in Figure 8, the standard deviation of density is calculated and reported in Figure 9(a). The density fluctuations are clearly maximum in the jet shear layer, and tend to be modulated along the shock-cell structure with a period of a shock-cell, which is consistent with previous experimental observations. ${ }^{23}$

The axial profile of the density fluctuations along a line located at the constant radius $r / D=0.63$ is given in Fig. 10(a). This profile presents maxima located between two consecutive shocks, which compares well with a similar profile presented in the figure 8 of reference 23. In the Figure 10(a) the axial profile of the maximum values of density fluctuations is presented. These are maximum upstream a shock-tip in the shear-layer and their level just downstream the shock is significantly lower than just upstream. Thus, the interactions of the turbulence in the jet shear layer with the shock-tip result in an inhibition of the growth of the level of density fluctuations, which is consistent with previous studies on velocity fluctuations. ${ }^{7,24}$

The standard deviation of the gray levels in the synthetic schlieren images are now considered. These images were obtained in the case where the (virtual) knife-edge is placed along the jet axis, and the gray levels are then noted $g_{y}$, and also in the case of a knife-edge placed orthogonal to the jet axis, the gray levels 


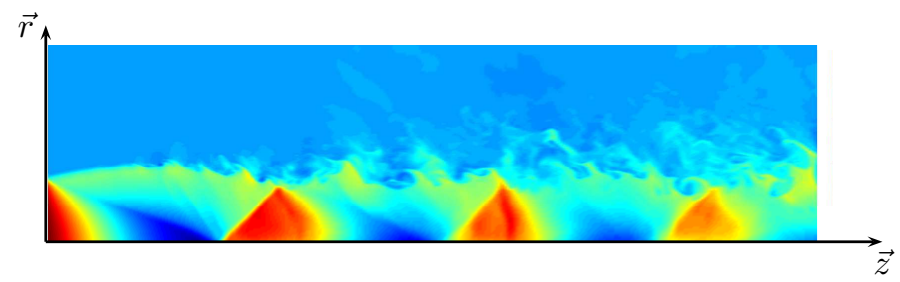

Figure 8. Density field of an underexpanded $M_{j}=1.56$ and $R e_{D}=5 \times 10^{4}$ jet, obtained by simulation ${ }^{16}$

(a)

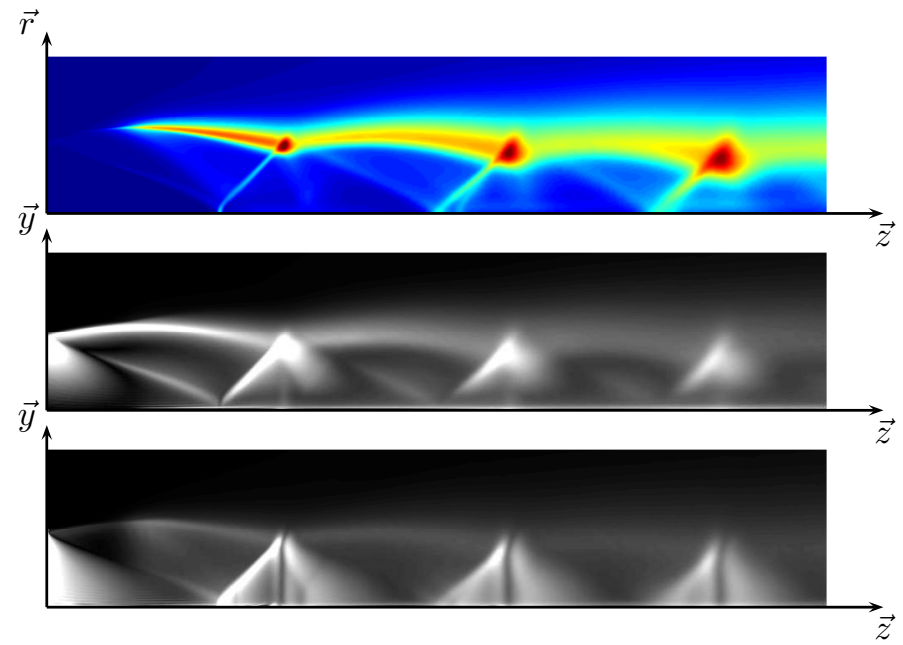

Figure 9. Maps of standard deviation of (a) density, (b) the gray level $g_{y}$ in the schlieren image with horizontal knife-edge $\left(f \equiv \frac{\partial \rho}{\partial y}\right)$, (c) the gray level $g_{z}$ in the schlieren image with vertical knife-edge $\left(f \equiv \frac{\partial \rho}{\partial z}\right)$. The colorbars in figures (b) and (c) are adjusted so that the maximum value is in white

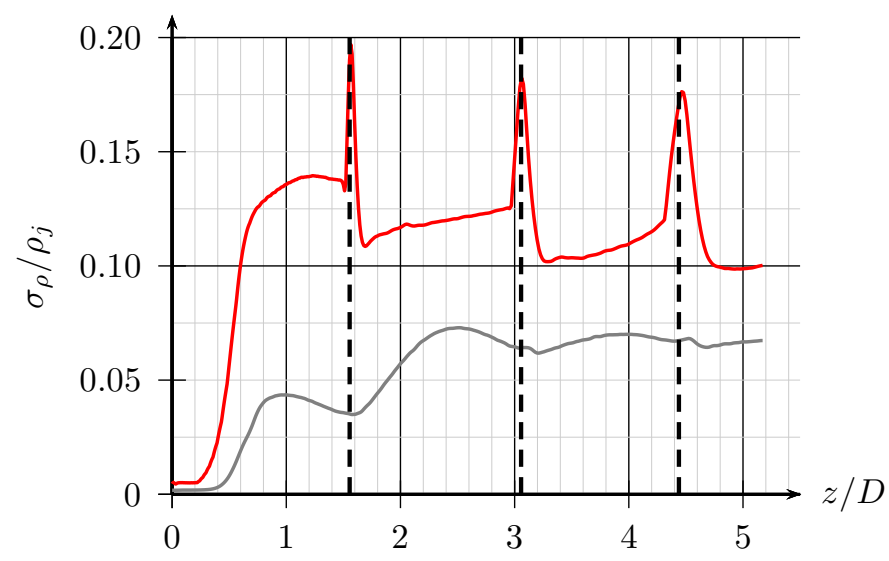

(a)

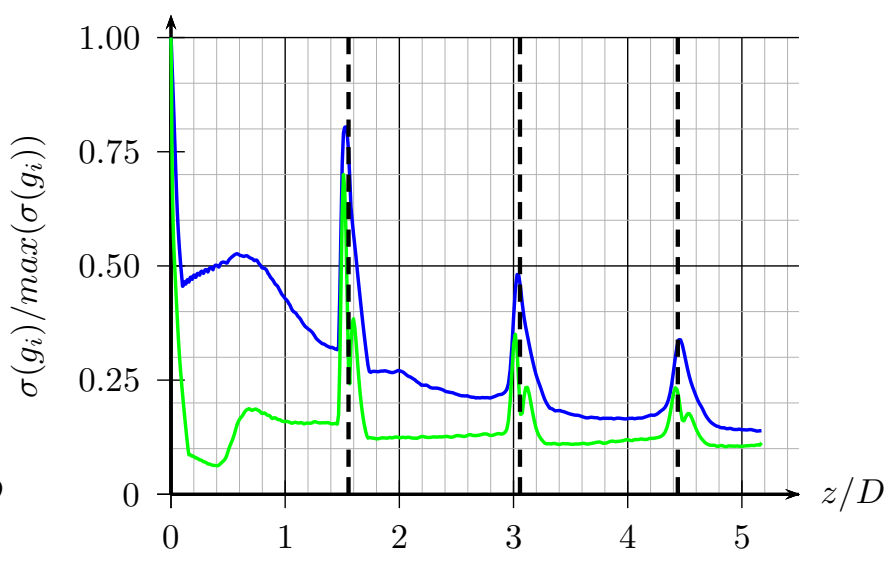

(b)

Figure 10. Axial evolution of (a) density fluctuations, taken along $-r / D=0.63$ and - the line of maximum density fluctuations, (b) the maximum of gray levels $g_{i}$ fluctuations, considering either schlieren images obtained with a knife-edge oriented along the jet axis $\left(g_{y},-\right)$ or normal to the jet axis $\left(g_{z},-\right)$. The dashed vertical lines correspond to the shock positions. 


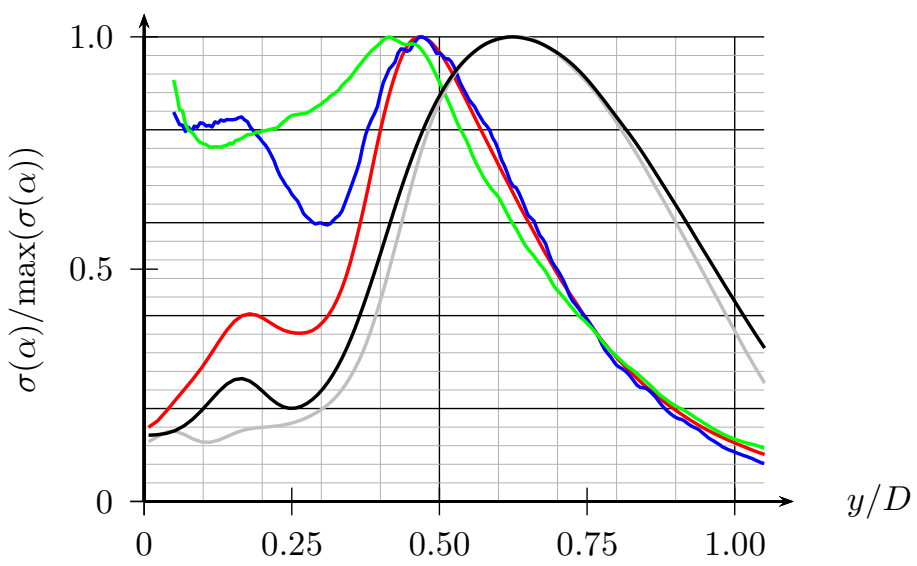

Figure 11. Radial profiles at axial distance $z_{0} / D=3.8$ of the standard deviation for different quantities $\alpha$ normalized by their maximum values; $\alpha \equiv-$ density, $-g_{y},-g_{z},-$ axial velocity, - radial velocity

being $g_{z}$. The maps of the standard deviations for $g_{y}$ and $g_{z}$ are given in Figure 9 (b) and (c), respectively. High levels of fluctuations for $g_{y}$ and $g_{z}$ are obtained in the areas of the schlieren image which correspond to

(i) the shear layer region, which is consistent with the presence of turbulence in this zone,

(ii) the inner part of the jet, and in particular near the shocks contained in the jet plume. This is the consequence of the integration of the optical disturbances over the light path. Thus, the fluctuations of $g_{y}$ and $g_{z}$ in this area can be viewed as a consequence of shock oscillations, especially encountered in screeching jets. ${ }^{21,25}$

Of interest here is to compare in the jet shear layer the results obtained with the flow density fluctuations. In Figure 10(b) the axial evolutions of the maximum value of the standard deviations for $g_{y}$ and $g_{z}$ are given. These evolutions are marked with the periodicity of the shock-cell structure, but the trend exhibited differs significantly from that of the maximum density fluctuations. For instance, the fluctuations in $g_{y}$ decrease between two consecutive shocks, and that of $g_{z}$ remain nearly constant, which is contrary to the axial evolution of $\sigma_{\rho}$.

On the other hand, the radial profiles of the fluctuations can be considered. They are illustrated in Figure 11 for the axial position $z_{0} / D=3.8$. The direct results from the simulation are the fluctuations of density, and of axial and radial velocity. Their profiles present a maximum value located in the inner part of the jet shear layer for the density fluctuations, and in its outer part for the velocity fluctuations. The radial profiles of the fluctuations of $g_{y}$ and $g_{z}$ are also shown and reach their maximum at a radial position close to that of maximum density fluctuations, in particular for $g_{y}$. As a conclusion for this point, even if the axial evolution of the maximum of density fluctuations is not comparable with the quantities extracted from schlieren images, the radial location of the maximum of density fluctuations in the jet shear layer seems to be found next to the position of the maximum of $g_{y}$ fluctuations.

\section{Convection velocity estimation}

The convection velocity is estimated using the phase of the cross-spectrum between two signals, as presented in section II.C. Two sets of data are considered here: density and schlieren images. In each case, pairs of time signals are extracted from either side of a reference point located at the axial position $z_{0} / D=3.8$. When using the density data, the radial position of the reference point is $r_{0} / D=0.45$, and when using the schlieren images, the position along the $\vec{y}$ axis is equivalently $y_{0} / D=0.45$. The signals include 1920 consecutive time-steps at the sampling frequency of $S t_{D}=6.4$ (see section II.B).

The phase of the cross-spectrum is computed between the signals extracted from the axial locations $z_{0}-\delta z$ and $z_{0}+\delta z$. An average of the results is obtained thanks to the jet axisymmetry: when using the density data, 512 cross-spectra are calculated around the jet axis by using the resolution in the azimuthal direction. Thus, the phase of the cross-spectrum is obtained using 512 blocks of 1760 time-steps. For the schlieren images, for a given time-step of the input density field, series of images are generated by rotating 


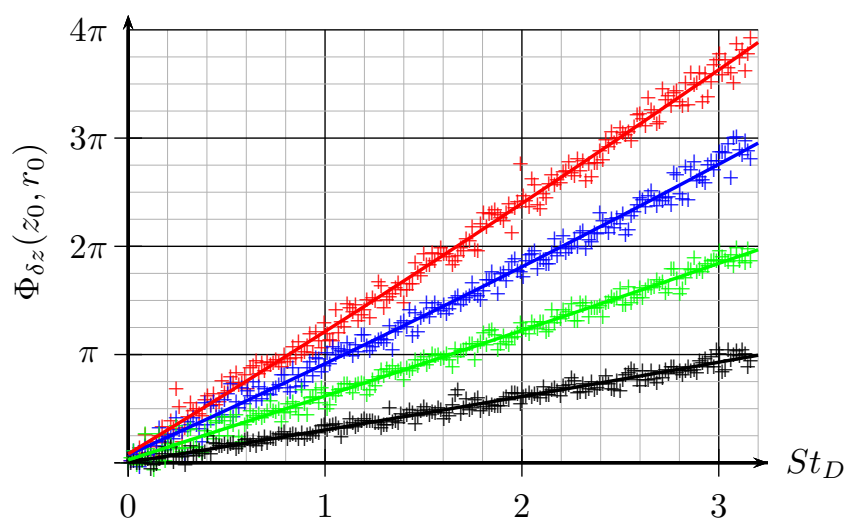

(a)

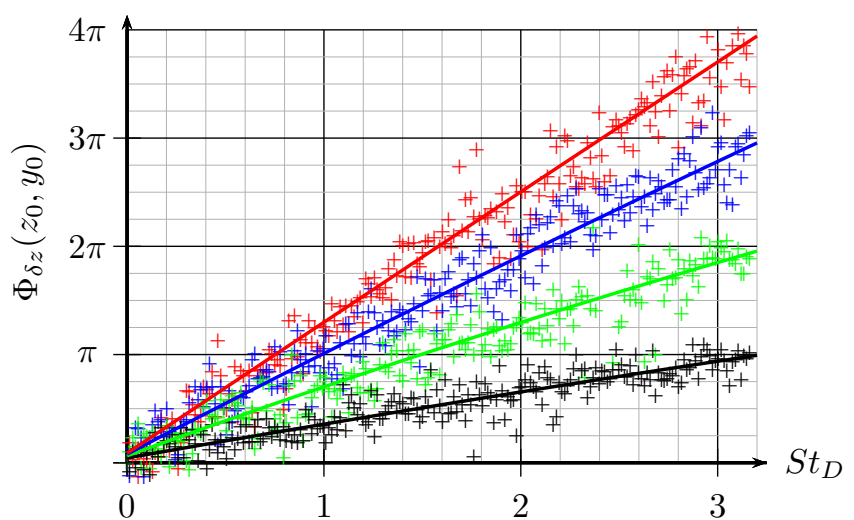

(b)

Figure 12. Phase evolution with Strouhal number for different separations between the two sensors monitoring (a) the density field, (b) the gray levels $g_{z}$ in the schlieren images. Separations $2 \delta_{z} / D:-\mathbf{0 . 1 2 1 ,} 0.241,-$ $0.362,-0.482$

the $(\vec{x}, \vec{y}, \vec{z})$ frame depicted in Figure 2 around the $\vec{z}$ axis. The number of rotations used to generate different schlieren images of the same flow could be 512, which would allow us to use the input density data without spatial interpolation. Nevertheless, because of spatial integration along the $\vec{x}$ axis, there is no obvious interest in choosing such a small angular difference between the schlieren images generated for a single time-step. Therefore, the number of rotations is limited here to 32. Thus, the phase of the cross-spectrum between schlieren data is obtained using 32 blocks of 1920 time-steps. It has been checked that this number of blocks, smaller than that used for the analysis of the density field, has a negligible influence on the results given below.

The phase of the cross-spectrum using both datasets is presented in Figure 12 as a function of $S t_{D}$. With the density signals, as presented in Figure 12(a), the phase evolution depends, as expected, on the separation between the two points where the data are extracted: the higher the separation, the faster the phase evolves. Considering the limited scatter of the results for one separation value, a second order polynomial fit of the data is used to represent for the dependency of $\Phi_{\delta z}$ with $S t_{D}$. These fits are superimposed on the Figure 12. The phases computed with schlieren data for different separation points are also represented in Figure 12(b), together with their polynomial fits. The trend is similar to that observed for density signals, with more scatter in particular for the large values of the separation $\delta z$. This scattering is attributed to the reduced coherence of the schlieren data with respect to the density data.

The convection velocity at the reference point is now estimated from the computed phase $\Phi_{\delta z}$. The results obtained with the density signals are presented in Figure 13 (a). The normalized convection velocity $U_{c} / U_{j}$ reaches values slightly above 0.75 in the high-frequency range. Furthermore, this value is almost independent of the separations $\delta z$, be it $0.241 D, 0.362 D$ or $0.482 D$, which is consistent with previous experimental results based on density measurements. ${ }^{3}$ For the smallest separation $2 \delta z=0.121 D, U_{c}$ differs, in the low frequency domain, from the other estimations probably because of the difficulty in capturing low frequency phenomena by monitoring two sensor points close one of each other. It can be noticed that the different estimations of the phase were obtained using the same methodology (same number of points, same polynomial-fit procedure) to avoid bias effects in data treatment.

The estimation of the convection velocity via the schlieren data is now considered. First, $U_{c} / U_{j}$ is calculated using the $g_{z}$ gray levels, which are those extracted from the synthetic schlieren images with $f \equiv \partial \rho / \partial z$ (knife-edge orthogonal to the jet axis), and the results are presented in Figure 13 (b). This case is interesting regarding the experimental results commented in section III, which corresponds to the same knife-edge setting. The general trend of $U_{c} / U_{j}$ increasing with $S t_{D}$ is well reproduced. By considering the results obtained for different probe spacings, one can point out a high-pass filtering behavior of the estimation of the convection velocity, with a cutoff frequency that decreases when the probe separation increases. The same effect is observed experimentally, as indicated by Figure 3, which indicates that this is inherent to the use of schlieren data, with the knife-edge orthogonal to the jet axis. This effect is less pronounced when considering the results obtained with the knife-edge along the jet axis, as visible in Figure 13 (c), 
(a)

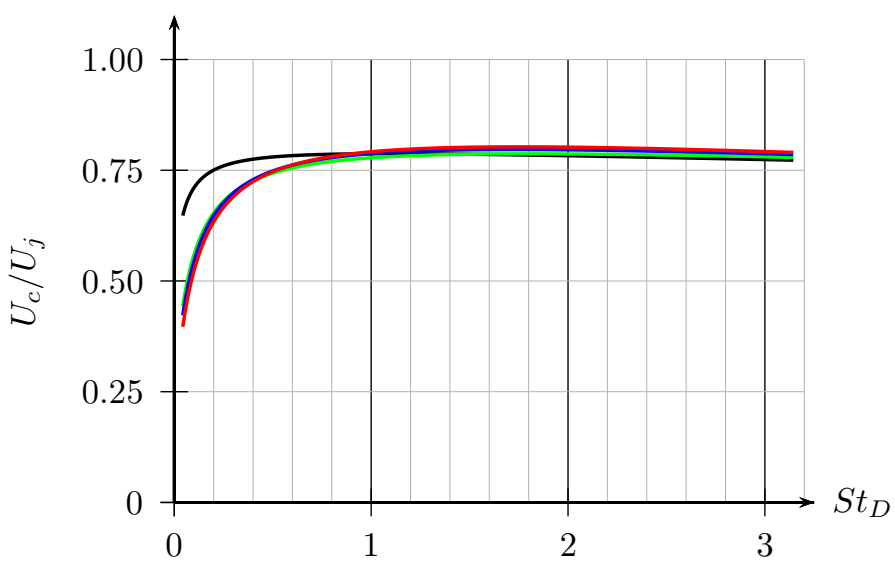

(b)

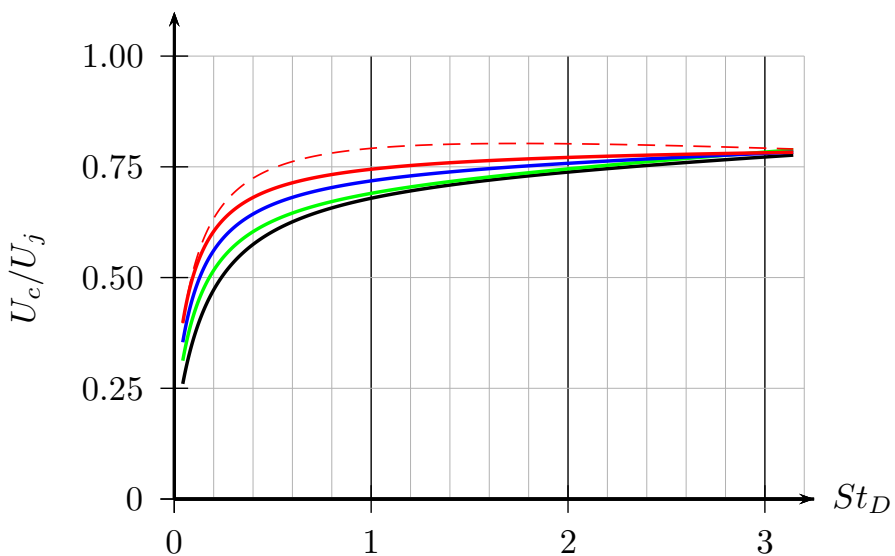

(c)

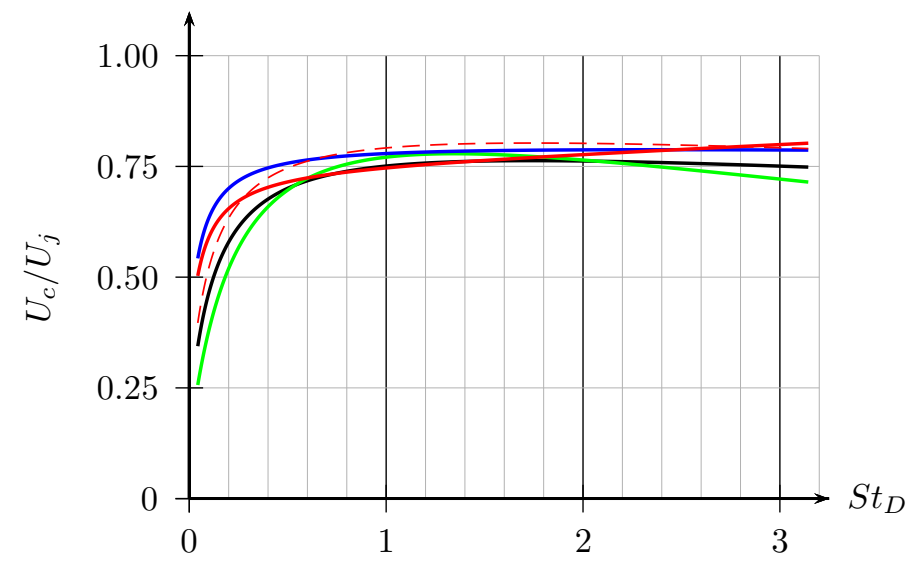

Figure 13. Convection velocity evolution with Strouhal number for different separations between the two sensors monitoring (a) the density field, (b) the gray levels $g_{z}$ from schlieren images, (c) the gray levels $g_{y}$ from schlieren images. Separations $2 \delta_{z} / D$ of $-0.121,-0.241,-0.362,-0.482$. In figures (b) and (c), the dashed curve corresponds to the result obtained with density signals. 
and the influence of the separation $\delta z$ appears less clear than in the other schlieren set-up. Using these data, averaging the results over the different separation points would lead to a reasonable estimation of the convection velocity.

\section{Conclusion}

A series of experimental schlieren images of an underexpanded $M_{j}=1.50$ jet are recorded at an acquisition frequency above $430 \mathrm{kHz}$ and with a knife-edge orthogonal to the jet axis. From the quantitative analyses of these data, the estimated convection velocity is found to be clearly affected by the axial distance between the two locations where the signals are extracted for the application of the phase shift method.

Thus, on the basis of the density field of an underexpanded jet determined numerically, the same kind of analysis is performed by comparing results inferred from the jet density field with the estimations from the processing of the schlieren images. These images are generated to mimic different knife-edge setups, oriented either along the jet axis or normal to it. The location of the maximum density fluctuations is found in a fair agreement with the location of the maximum of gray levels fluctuations in the schlieren images, in particular if the (virtual) knife-edge is set orthogonal to the jet axis. For the convection velocity estimation, it confirms the bias error due to separation between the two probes, which leads an underestimation of the convection velocity for low-frequencies in particular when the sensitivity of the schlieren images relies on axial density gradients. This trend is consistent with the conclusion drawn from the experimental study. It is of interest to determine, by further analyses, whether such a trend comes from the effects of space integration along the light beam or from the effects of examining the density gradients rather than the density itself.

\section{Acknowledgments}

This work was performed within the framework of the Labex CeLyA of Université de Lyon, within the program Investissements d'Avenir (ANR-10-LABX-0060/ ANR-11-IDEX-0007) operated by the French National Research Agency (ANR).

\section{References}

\footnotetext{
${ }^{1}$ Raman, G., "Supersonic jet screech : half-century from Powell to the present," Journal of Sound and Vibration, Vol. 225, No. 3, 1999, pp. $543-571$.

${ }^{2}$ Tam, C. K. W., "Supersonic Jet Noise," Annual Review of Fluid Mechanics, Vol. 27, No. 1, 1995, pp. 17-43.

${ }^{3}$ Panda, J., "Two Point Space-Time Correlation of Density Fluctuations Measured in High Velocity Free Jets," AIAA2006-0006, 2006.

${ }^{4}$ Kerhervé, F., Jordan, P., Gervais, Y., and Valière, J.-C., "Aerodynamic characterization of a supersonic jet using twopoint laser Doppler velocimetry," AIAA 2003-3215, 2003.

${ }^{5}$ Wernet, M. P., "Temporally resolved PIV for space-time correlations in both cold and hot jet flows," Measurement Science and Technology, Vol. 18, No. 5, 2007, pp. 1387-1403.

${ }^{6}$ Veltin, J., On the Characterization of Noise Sources in Supersonic Shock Containing Jets, Ph.D. thesis, Pennsylvania State University, 2008.

${ }^{7}$ André, B., Étude expérimentale de l'effet du vol sur le bruit de choc de jets supersoniques sous-détendus, $\mathrm{Ph} . \mathrm{D}$. thesis, No.2012-42, École Centrale de Lyon, 2012.

${ }^{8}$ Mercier, B., Castelain, T., and Bailly, C., "Investigation of under-expanded jet screech associated convective velocity based on high frequency sampled schlieren visualisations," 22ème Congrès Franais de Mécanique, Lyon,, 2015.

${ }^{9}$ Doty, M. and McLaughlin, D., "Space-time correlation measurements of high-speed axisymmetric jets using optical deflectometry," Experiments in Fluids, Vol. 38, 2005, pp. 415-425.

${ }^{10}$ Kuo, C., Power, R., and McLaughlin, D., "Space-Time Correlation of Flow and Acoustic Field Measurements in Supersonic Helium-Air Mixture Jets Using Optical Deflectometry," AIAA 2011-2789, 2011.

${ }^{11}$ Weinstein, L., "Large-Field High-Bightness Focusing Schlieren System," AIAA J., Vol. 31, 1993, pp. 1250-1255.

${ }^{12}$ Taghavi, R. and Raman, G., "Visualization of supersonic screeching jets using a phase conditioned focusing schlieren system," Experiments in Fluids, Vol. 20, 1996, pp. 472-475.

${ }^{13}$ Bogey, C. and Bailly, C., "A family of low dispersive and low dissipative explicit schemes for noise computation," Journal of Computational Physics, Vol. 194(1), 2004, pp. 194-214.

${ }^{14}$ Berland, J., Bogey, C., and Bailly, C., "A study of differentiation errors in large-eddy simulations based on EDQNM theory," Journal of Computational Physics, Vol. 227(18), 2008, pp. 8314-8340.

${ }^{15}$ Bogey, C., de Cacqueray, N., and Bailly, C., "A shock-capturing methodology based on adaptative spatial filtering for high-order non-linear computations," Journal of Computational Physics, Vol. 228(5), 2009, pp. 1447-1465.

${ }^{16}$ Gojon, R., Bogey, C., and Marsden, O., "Large-eddy simulation of underexpanded round jets impinging on a flat plate 4 to 9 radii downstream from the nozzle," 21st AIAA/CEAS Aeroacoustics Conference, Dallas, TX, 2015.
} 
${ }^{17}$ McIntyre, S. and Settles, G., "Optical Experiments on Axisymmetric Compressible Turbulent Mixing Layers," AIAA 91-0623, 1991.

${ }^{18}$ Emrich, R., editor, Methods in Experimental Physics Volume 18, Part A, Fluid Dynamics, Academic Press, 1981.

${ }^{19}$ Merzkirch, W., Flow Visualization, Academic Press, 1987.

${ }^{20}$ Weyl, F., Physical measurements in gas dynamics and combustion, Princeton University Press, 1954.

${ }^{21}$ Panda, J., "Shock oscillation in underexpanded screeching jets," Journal of Fluid Mechanics, Vol. 363, 1998, pp. 173-198.

${ }^{22}$ André, B., Castelain, T., and Bailly, C., "Shock oscillations in a supersonic jet exhibiting antisymmetrical screech," AIAA J., Vol. 50, 2012, pp. 2017-2020.

${ }^{23}$ Panda, J. and Seasholtz, R. G., "Measurement of shock structure and shock-vortex interaction in underexpanded jets using Rayleigh scattering," Phys. Fluids, Vol. 11, No. 12, 1999, pp. 3761-3777.

${ }^{24}$ Gojon, R., Étude de jets supersoniques impactant une paroi par simulation numérique. Analyse aérodynamique et acoustique des mécanismes de rétroaction, Ph.D. thesis, No.2015-39, École Centrale de Lyon, 2015.

${ }^{25}$ André, B., Castelain, T., and Bailly, C., "A shock-tracking procedure for studying screech-induced oscillations," AIAA J., Vol. 49, 2011, pp. 1563-1566. 